\title{
Hardy's Condition in the Moment Problem for Probability Distributions
}

\author{
Jordan Stoyanov ${ }^{1}$ and Gwo Dong $\operatorname{Lin}^{2,3}$ \\ ${ }^{1}$ Newcastle University, Newcastle upon Tyne, UK \\ 2 Academia Sinica, Taipei, TAIWAN \\ ${ }^{3}$ Corresponding author: Gwo Dong Lin, e-mail: gdlin@stat.sinica.edu.tw
}

\begin{abstract}
In probabilistic terms Hardy's condition is written as follows: $E\left[e^{c \sqrt{X}}\right]<\infty$, where $X$ is a nonnegative random variable and $c>0$ a constant. If this holds, then all moments of $X$ are finite and the distribution of $X$ is uniquely determined by the moments. This condition, based on two papers by G. H. Hardy $(1917 / 1918)$, is weaker than Cramér's condition requiring the existence of a moment generating function of $X$. We elaborate Hardy's condition and show that the constant $\frac{1}{2}$ (square root) is the best possible for the moment determinacy of $X$. We describe relationships between Hardy's condition and properties of the moments of $X$. We use this new condition to establish a result on the moment determinacy of an arbitrary multivariate distribution.
\end{abstract}

Keywords: moments, moment problem, Hardy's condition, Cramér's condition, Carleman's condition

\section{Introduction}

A web-search for 'Hardy's condition in the moment problem for probability distributions' shows that nothing is available. The reader is invited to check and eventually confirm that this is the case.

In this paper we provide details about a not so well-known criterion for uniqueness of a probability distribution by its moments. The criterion is based on two old papers by G. H. Hardy, and to the best of our knowledge it is not explicitly described in the probabilistic literature. Hardy's condition has a simple form, often it is relatively easy to check and it allows us to derive new results or provide new proofs of known results.

Hardy's criterion is intrinsically related to other well-known classical criteria based on conditions of Cramér, Carleman, Krein (see, e.g., Shiryaev (1996), Stoyanov (1997)) and also to more recent developments achieved in Lin (1997), Stoyanov (2000), Pakes (2001), Pakes et al. (2001), De Jeu (2003) and Stoyanov (2004).

G. H. Hardy (1877-1947), who is recognized as one of the greatest analysts of 20th century, never showed an interest in probability theory, never tried to solve a probabilistic problem and never used probabilistic language. However, in a recent paper, P. Diaconis (2002) convincingly concluded that several analytical results by G. H. Hardy have been instrumental later when building some areas of modern probability theory. Diaconis did not refer to the two papers by Hardy which are in the base of the present paper. The new criterion described here shows that in fact the analytical results in Hardy $(1917,1918)$, though not intended to be probabilistic, are, when appropriately reformulated and annotated, definitely another contribution to probability theory, in particular, to the moment problem for probability distributions.

\section{Notations, notions and preliminary facts}

Suppose that $X$ is a random variable with values on the whole real line $\mathbb{R}^{1}$. If $F$ 
denotes the distribution function of $X$, we write $X \sim F$. We assume that $X$ has all moments finite, i.e. $E\left[|X|^{k}\right]<\infty$ for $k=1,2, \ldots$, and use the notation $m_{k}$ for the moment of order $k$ :

$$
m_{k}=E\left[X^{k}\right]=\int_{\mathbb{R}^{1}} x^{k} d F(x), k=1,2, \ldots
$$

Thus the moment sequence $\left\{m_{k}, k=1,2, \ldots\right\}$ of $X$, and $F$, is well-defined. If $F$ is the only distribution function with the moments $\left\{m_{k}\right\}$, we say that $F$ is uniquely determined by its moments, or that $F$ (or $X$ ) is $M$-determinate. Otherwise $F$ is nonunique, or $F$ (or $X$ ) is $M$-indeterminate in which case there is at least one distribution function, say $G$, such that $F \neq G$, but $m_{k}(F)=m_{k}(G)$ for all $k=1,2, \ldots$

According to well-known Cramér's criterion, if $X$ is an arbitrary random variable, $X \sim F$, whose moment generating function exists, i.e. for some $t_{0}>0$, the function $M(t)=E\left[e^{t X}\right]<\infty$ for all $t \in\left(-t_{0}, t_{0}\right)$ (Cramér's condition), then all moments of $X$ are finite, and $F$ is M-determinate. If there is no moment generating function, i.e. $E\left[e^{t_{n} X}\right]=\infty$ for some points $t_{n}$ converging to zero, we say that $F$ has heavy tail(s). Still, we may have all moments $m_{k}$ finite and $F$ either M-determinate or M-indeterminate.

There are criteria for uniqueness and for non-uniqueness of a distribution in terms of the moments. They depend on the range of values of $X, X \sim F$, equivalently, on the support of $F$. Two cases are of importance, when the support is a subset of the positive semi-real line $\mathbb{R}^{+}=[0, \infty)$ (Stieltjes moment problem), and when the support is a subset of the whole real line $\mathbb{R}^{1}=(-\infty, \infty)$ (Hamburger moment problem).

It is possible that a distribution $F$ on $\mathbb{R}^{+}$is unique for the Stieltjes moment problem, but non-unique for the Hamburger moment problem. This can eventually happen only for discrete distributions with unbounded support which includes the origin; for details see Chihara (1968), p. 481. In this paper we focus our attention on the Stieltjes moment problem.

\section{Results}

We are now in a position to formulate the results discussed in this paper.

Theorem 1 (Hardy's Criterion). Let $X$ be a nonnegative random variable with distribution function $F$. Suppose that the following condition is satisfied for some $c>0$ :

$$
E\left[e^{c \sqrt{X}}\right]<\infty \quad \text { (Hardy's condition). }
$$

Then all positive integer order moments of $X$ are finite, i.e. $m_{k}=E\left[X^{k}\right]=$ $\int_{0}^{\infty} x^{k} d F(x)<\infty, k=1,2, \ldots$, and moreover, $F$ is M-determinate, i.e. $F$ is the only distribution function with the moment sequence $\left\{m_{k}, k=1,2, \ldots\right\}$.

Remark. Let us emphasize that the required condition is on $\sqrt{X}$, i.e. we require the $\frac{1}{2}$-Cramér's condition for $X$, or Cramér's condition for $\sqrt{X}$. This implies that $\sqrt{X}$ is M-determinate. However, the conclusion in the theorem is about $X$ itself. Equivalently, under Hardy's condition, $F$ is the only distribution function with the moment sequence $\left\{m_{k}, k=1,2, \ldots\right\}$.

Corollary 1. If the random variable $X \geq 0$ satisfies Cramér's condition $E\left[e^{c X}\right]<\infty$ for some $c>0$, then $X^{2}$ is M-determinate.

Corollary 2. If $X \geq 0$ is a random variable with $E\left[\exp \left(c X^{\delta}\right)\right]<\infty$ for some $c>0$, then the transformed random variable $X^{2 \delta}$ is M-determinate.

Theorem 2. The constant $\frac{1}{2}$ (square root) in Hardy's condition (1) is the best possible. In other words, for each $\rho \in\left(0, \frac{1}{2}\right)$, there exists a random variable $X \geq 0$ such that $E\left[e^{c X^{\rho}}\right]<\infty$ for some $c>0$, however $X$ is M-indeterminate. 
Theorem 3. Let $a>0$ and $X \sim F$ be a nonnegative random variable.

(i) If $E\left[e^{c X^{a}}\right]<\infty$ for some constant $c>0$, then $m_{k} \leq \Gamma(k / a+1) c_{0}^{k}, k=1,2, \ldots$, for some constant $c_{0}>0$ (independent of $k$ ).

(ii) Conversely, if, in addition, $a \leq 1$, and $m_{k} \leq \Gamma(k / a+1) c_{0}^{k}, k=1,2, \ldots$, for some constant $c_{0}>0$ (independent of $k$ ), then $E\left[e^{c X^{a}}\right]<\infty$ for some $c>0$.

Corollary 3. Let $X \sim F$ be a nonnegative random variable. Then:

(i) $E\left[e^{c \sqrt{X}}\right]<\infty$ for some $c>0$ if and only if $m_{k} \leq(2 k) ! c_{0}^{k}, k=1,2, \ldots$, for some $c_{0}>0$

(ii) $E\left[e^{c X}\right]<\infty$ for some $c>0$ if and only if $m_{k} \leq k ! c_{0}^{k}, k=1,2, \ldots$, for some $c_{0}>0$.

The moment condition in Theorem 3 has the following equivalent form.

Theorem 4. Let $a>0$ and $X \sim F$ be a nonnegative random variable. Then $\limsup _{k \rightarrow \infty} \frac{1}{k} m_{k}^{a / k}<\infty$ if and only if $m_{k} \leq \Gamma(k / a+1) c_{0}^{k}, k=1,2, \ldots$, for some $c_{0}>0$ (independent of $k$ ).

Corollary 4. Let $a \in(0,1]$ and $X \sim F$ be a nonnegative random variable. Then $E\left[e^{c X^{a}}\right]<\infty$ for some $c>0$ if and only if $\limsup _{k \rightarrow \infty} \frac{1}{k} m_{k}^{a / k}<\infty$.

Corollary 5. Let $X \sim F$ be a nonnegative random variable whose moments satisfy the condition $\lim \sup _{k \rightarrow \infty} \frac{1}{k} m_{k}^{1 / k}<\infty$. Then $X^{2}$ is M-determinate.

The following surprising result demonstrates the power and usefulness of Hardy's condition.

Theorem 5. Let $Y, Y \sim G$, be an arbitrary random variable on $\mathbb{R}^{1}$ satisfying Cramér's condition, i.e. the moment generating function of $Y$ exists. Then:

(i) The random variable $Y^{2}$ is M-determinate on $\mathbb{R}^{+}$.

(ii) If, in addition, $G$ does not have a mass at the origin, the random variable $Y^{2}$ is M-determinate on $\mathbb{R}^{1}$.

Corollary 6. If $X>0$ satisfies Cramér's condition, then $X^{2}$ is M-determinate on $\mathbb{R}^{+}$and also on $\mathbb{R}^{1}$.

While the problem of moments for one-dimensional distributions is in general well developed and several results are available in the literature, very little is done for multivariate distributions. The paper Kleiber and Stoyanov (2013) summarizes known analytical results and contains some new results. Now, having to hand Hardy's criterion, we can establish a new and quite general result.

Suppose $X=\left(X_{1}, \ldots, X_{n}\right)$ is an arbitrary random vector in $n$-dimensional Euclidean space $\mathbb{R}^{n}$ with all multi-indexed moments

$$
m_{k_{1}, \ldots, k_{n}}=E\left[X_{1}^{k_{1}} \cdots X_{n}^{k_{n}}\right], k_{j}=1,2, \ldots, j=1,2, \ldots, n,
$$

finite. We are interested in the determinacy of the vector $X \in \mathbb{R}^{n}$ by the multiindexed moment sequence $\left\{m_{k_{1}, \ldots, k_{n}}, k_{j}=1,2, \ldots, j=1,2, \ldots, n\right\}$. Since the distribution of $X$ is arbitrary, this is a Hamburger moment problem.

Denote by $\|X\|$ the Euclidean length of $X$, i.e.

$$
\|X\|=\sqrt{\|X\|^{2}}=\sqrt{X_{1}^{2}+\cdots+X_{n}^{2}} .
$$

Theorem 6. With the notations introduced above, suppose that the one-dimensional nonnegative random variable $\|X\|$ satisfies Cramér's condition:

$$
E\left[e^{c\|X\|}\right]<\infty \text { for some } c>0 .
$$


Then the random vector $X \in \mathbb{R}^{n}$, or equivalently, its $n$-dimensional distribution function, is uniquely determined by the set of multi-indexed moments $\left\{m_{k_{1}, \ldots, k_{n}}, k_{j}=\right.$ $1,2, \ldots, j=1,2, \ldots, n\}$.

\section{Acknowledgment}

We would like to express our thanks to Professor Nick Bingham for his constructive comments and suggested additional references.

\section{References}

Akhiezer, N. I. (1965). The Classical Problem of Moments and Some Related Questions of Analysis. Oliver \& Boyd, Edinburgh. [Original Russian edition, Nauka, Moscow, 1961.]

Carleman, T. (1926). Les Fonctions Quasi-analytiques. Gauthier-Villars, Paris.

Chihara, T. S. (1968). On indeterminate Hamburger moment problems. Pacific J. Math., 27, 475-484.

De Jeu, M. (2003). Determinate multidimensional measures, the extended Carleman theorem and quasi-analytic weights. Ann. Probab., 31, 1205-1227.

Diaconis, P. (2002). G. H. Hardy and probability??? Bull. London Math. Soc., 34, $385-402$.

Hardy, G. H. (1917). On Stieltjes' 'problème des moments'. Messenger of Math., 46, 175-182. [Collected Papers of G. H. Hardy. Volume VII, pp. 75-83. Oxford Univ. Press, Oxford, 1979.]

Hardy, G. H. (1918). On Stieltjes' 'problème des moments' (continued). Messenger of Math., 47, 81-88. [Collected Papers of G. H. Hardy. Volume VII, pp. 84-91. Oxford Univ. Press, Oxford, 1979.]

Kleiber, C. and Stoyanov, J. (2013). Multivariate distributions and the moment problem. J. Multivar. Analysis, 113, 7-18.

Lin, G. D. (1997). On the moment problems. Statist. Probab. Letters, 35, 85-90. Erratum, ibid, 50(2000), 205.

Pakes, A. G. (2001). Remarks on converse Carleman and Krein criteria for the classical moment problem. J. Austral. Math. Soc., 71, 81-104.

Pakes, A. G., Hung, W.-L. and Wu, J.-W. (2001). Criteria for the unique determination of probability distributions by moments. Austral. N. Z. J. Statist., 43, $101-111$.

Putinar, M. and Schmüdgen, K. (2008). Multivariate determinateness. Indiana Univ. Math. J., 57, 2931-2968.

Shiryaev, A. N. (1996). Probability. 2nd ed. Springer, Berlin.

Stoyanov, J. (1997). Counterexamples in Probability. 2nd edn. John Wiley \& Sons, Chichester.

Stoyanov, J. (2000). Krein condition in probabilistic moment problems. Bernoulli, 6, 939-949.

Stoyanov, J. (2004). Stieltjes classes for moment-indeterminate probability distributions. J. Appl. Probab., 41A, 281-294. 